\title{
Spectrum of Endometrial lesions in patients presenting with abnormal uterine bleeding
}

\author{
M Banyameen Iqbal ${ }^{1}$, Tushar Kambale ${ }^{2, *}$, Anushree Khandelwal ${ }^{3}$, Ashily Koshy ${ }^{4}$, Bedarshi Banerjee ${ }^{5}$ \\ 1,2 Associate Professor, ${ }^{\mathbf{3}, 4,5}$,Resident, Dept. of Pathology, Dr. D.Y Patil Medical College, Hospital and Research Centre, Pimpri, \\ Pune, Maharashtra, India \\ *Corresponding Author: Tushar Kambale \\ Email: drtusharkambale@gmail.com
}

Received: $25^{\text {th }}$ April, 2018

Accepted: $29^{\text {th }}$ May, 2018

\begin{abstract}
Introduction: Most of the patients with bleeding per vaginum undergo either colposcopic evaluation or Pap smear for cervical pathology or Trans Vaginal Ultrasound and endometrial biopsy to diagnose the definite pathology affecting endometrium. This study was done to see the pattern of histopathological spectrum of endometrial biopsy and their clinical correlation in a tertiary care hospital.

Materials and Methods: This was a retro-prospective study conducted at a tertiary care hospital in western Maharashtra. 1000 biopsy cases were studied. Light microscopy examination and clinical diagnoses were correlated.

Results: out of 1000 patient's maximum number belonged to perimenopausal age group followed by reproductive age group and then postmenopausal age group. In 483 cases normal histopathological pattern was observed, out of which 259 cases showed proliferative phase endometrium (25.9\%) and 224 cases showed secretory phase $(22.4 \%)$. 104 cases showed simple (10.4\%) and 43 cases (4.3\%) complex hyperplasia. Exogenous hormonal effect was seen in 148 cases (14.8\%). The least pattern which was observed was endometrial carcinoma, seen in only $2.3 \%$ of patients.

Conclusion: The study showed that carcinoma was most common in the post-menopausal age group. This group also had the maximum frequency of atrophic endometrium and complex hyperplasia. Proliferative endometrium was recorded highest in perimenopausal age group while reproductive age group showed highest frequency of secretory type endometrium. This study is in concordance with most other conducted studies on similar parameters.
\end{abstract}

Keywords: Biopsy, Endometrium, Uterine bleeding.

\section{Introduction}

Excessive and irregular bleeding is still considered to be one of the most common presenting symptom in Gynaecological practice amongst all age group of patients. ${ }^{1}$ Abnormal uterine bleeding may be defined as a bleeding pattern that differs in frequency, duration, and amount from a pattern observed during a normal menstrual cycle or after menopause. ${ }^{2}$ It includes both dysfunctional uterine bleeding (DUB) and bleeding due to identifiable causes like endocrine and /or psychological cause and also bleeding from structural causes like fibroids, polyps, endometrial carcinoma, and pregnancy complications.

Dysfunctional Uterine bleeding is a condition where bleeding cannot be attributed to any identifiable organic pathology in the endometrium. High levels of oestrogen in circulating blood cause the same. ${ }^{3-5}$

The cytological studies include either brush or jet washing, but their efficacy in determining the malignant potential of a lesion yet remains unsatisfactory owing to artifactual changes during processing. ${ }^{6}$ The idea of suction and curettage was first introduced by Novak and Randall and this study was undertaken to determine the spectrum of various endometrial lesions determined by HPE and determine their clinico-pathological correlation.

\section{Materials and Methods}

The present study was conducted at department of Pathology in a tertiary care hospital in western Maharashtra. It was a retrospective analysis of 1000 endometrial biopsy specimens received from Gynaecology department over a period extending from January 2014 to June 2017. Samples were taken from patients who had presented with abnormal uterine bleeding.

The patients were subdivided into six groups according to the pattern of abnormal uterine bleeding: ${ }^{5}$

1. Polymenorrhoea - frequent episodes of menstruation, usually occurring at intervals of 21 days or less.

2. Oligomenorrhoea - denotes infrequent, irregularly timed episodes of bleeding usually occurring at intervals of more than 35 days.

3. Menorrhagia - regularly timed episodes of bleeding that are excessive in amount $(80 \mathrm{~mL})$ and/or duration of flow (5 days).

4. Metrorrhagia - irregularly timed episodes of bleeding superimposed on normal cyclical bleeding.

5. Menometrorrhagia- means excessive, prolonged bleed.

6. Post-menopausal bleeding.

Ethical committee clearance was obtained.

Endometrial tissue was collected by sampling 
procedures such as dilatation and curettage (D\&C) and fractional curettage among others. Endometrial tissues received were in $10 \%$ Formalin solution in containers appropriately labelled for name, age and type of specimen. Specimens were fixed overnight and then processed, sectioned and stained with Harris's Haematoxylin and Eosin and microscopic evaluation was done. Immunohistochemical staining was done as applicable. Detailed clinical history and relevant investigations done were also documented. Specimens received as products of conception and inadequate specimens comprising mainly of blood clots and mucous were excluded from the study. Statistical tests were applied wherever required.

\section{Result}

During the study period, out of 1000 patients most patients belonged to the perimenopausal age group i.e. between 40-50 years (424) followed by the reproductive age group $<40$ years $(400)$ and then the postmenopausal age group $>50$ years (176).

Patients were distributed according to pattern of bleeding. Maximum numbers of patients were presented as menorrhagia 360 cases $(36 \%)$ followed by polymenorrhoea 204 cases $(20.40 \%)$. Postmenopausal bleeding, metrorrhagia and oligomenorrhoea were seen in 171 cases (17.1\%), 16.70\% (167 cases) and, $6.0 \%$ (60 cases) respectively. Minimum numbers of patients presented with menometrorrhagia 38 cases $(3.80 \%)$ (Fig. 1).

Histopathological evaluation of all biopsies revealed various patterns including endometrial polyp, endometritis, atrophic changes, carcinoma, hyperplasia etc. In 483[48.3\%] cases normal histopathological pattern was observed, out of which 281[28.1\%], cases showed proliferative phase endometrium whereas in 202 [20.2\%] cases secretory phase was noted. In $147[14.7 \%$ ] cases hyperplastic endometrium was observed. Among the hyperplastic cases 108[10.8\%] were simple and complex in the remaining $39(3.9 \%)$ cases. Exogenous hormonal effect was seen in 148 cases $(14.8 \%)$. Other patterns were seen with less frequency i.e. $<10 \%$. The least pattern which was observed was endometrial carcinoma, seen in only 23 [2.3\%] of patients. [Fig. 2, 3, 4]

Stratification by age was done and frequencies of different endometrial histopathological patterns were observed in the various age groups. In $<40$ years age group, out of 400 cases, secretory phase was observed in 130 [32.5\%] of cases and was the most frequent, whereas proliferative phase was seen in $27 \%$ of cases (108). Carcinoma was seen in 4 [1.0\%] cases while atrophic endometrium was not reported. Other patterns reported are shown in [Table 1,2]

Among 424 cases in 40-50 years age group the most frequent pattern observed was proliferative, seen in $119(28.06 \%)$ cases followed by exogenous hormonal effect in 87 cases $(20.5 \%)$. Carcinoma was seen in 8 cases $(1.8 \%)$. Other patterns reported are shown in [Table 1,2]

In > 50 years age group, maximum pattern observed was atrophic endometrium in 51 cases (28.9\%) followed by proliferative phase in 32 cases (18.18\%). Carcinoma was reported in $11(6.25 \%)$ cases. No case of endometritis was documented. Other patterns reported are shown in [Table 1,2]

Table shows clinical presentation and histopathological findings. Of all the 360 cases of menorrhagia the most frequent pattern observed was normal endometrium in $203(56.3 \%)$ cases, followed by exogenous hormonal effect seen in $62(17.2 \%)$ cases. Endometrial carcinoma was seen in $4(1.1 \%)$ cases whereas atrophic pattern was not observed in any of the biopsy specimen. [Table 1, 2]

In 204 cases of polymenorrhoea $105(51.4 \%)$ cases were normal whereas only $1(0.49 \%)$ case of carcinoma was seen. Out of 167 cases of metrorrhagia 122 (73.0\%) were normal and only $4(2.39 \%)$ cases were showing carcinoma. [Table 1,2]

In 171 cases of postmenopausal bleeding hyperplasia was the most common pattern observed in $73(42.6 \%)$ cases followed by atrophic pattern in $42(24.5 \%)$ cases. Carcinoma was seen in $11(6.43 \%)$ cases. [Table 1,2]

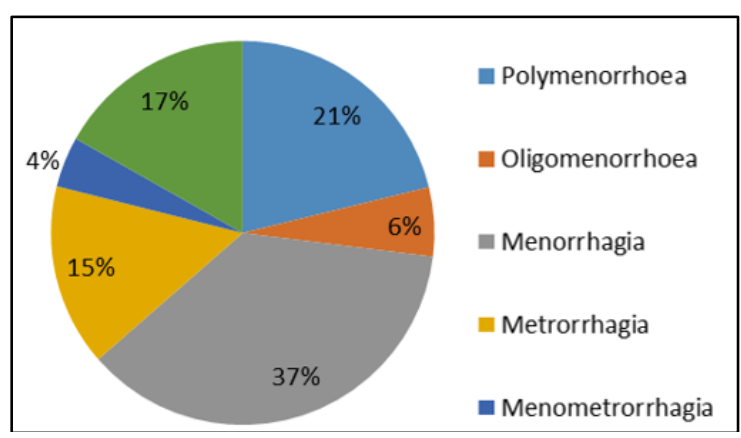

Fig. 1: Distribution of cases according to types of abnormal uterine bleeding

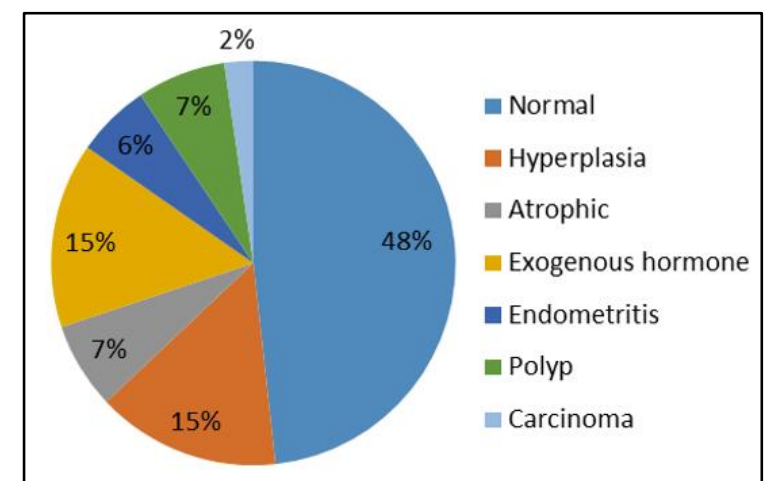

Fig. 2: Distribution of Histopathologic diagnosis of 1000 endometrial biopsies. 

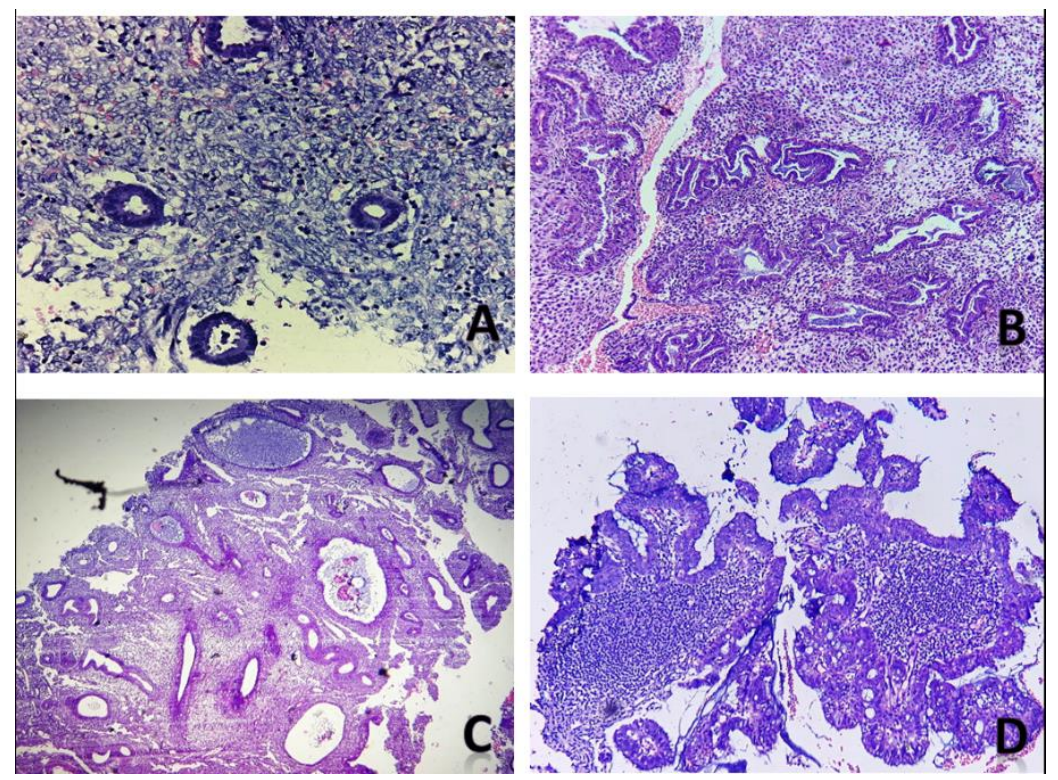

Fig. 3: (A) proliferative endometrium; (B) Secretory endometrium; (C) Simple cystic hyperplasia; (D) Complex atypical hyperplasia
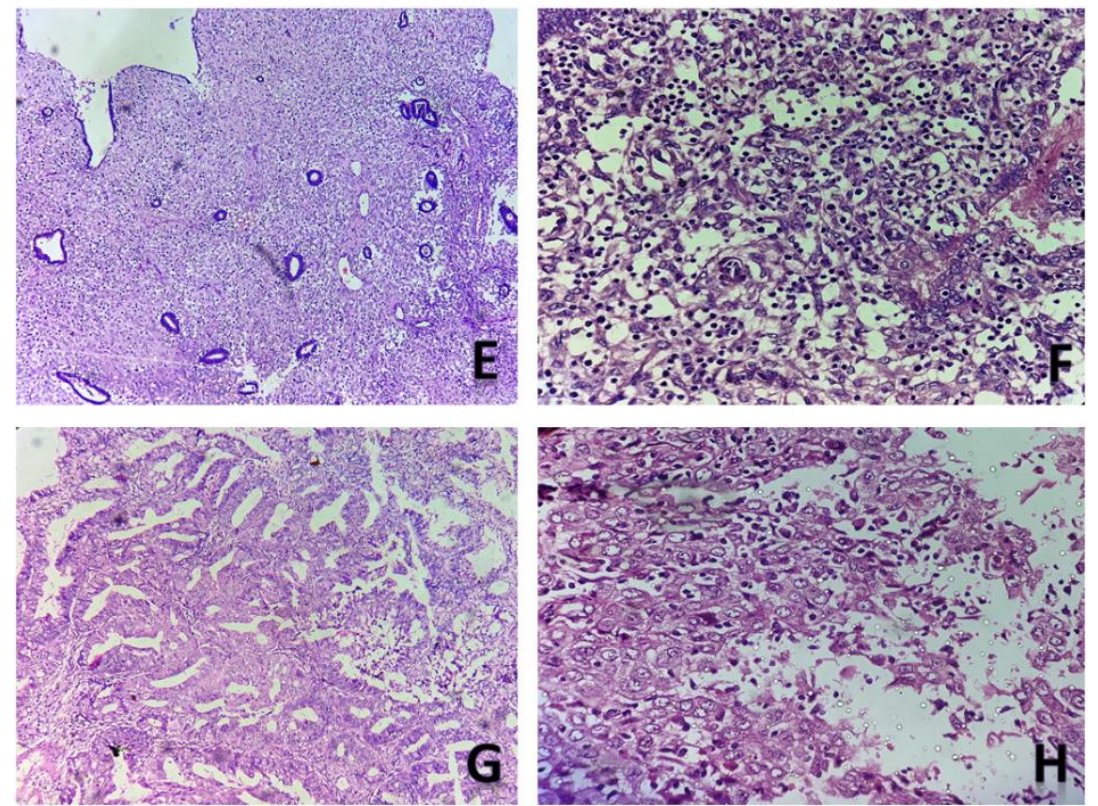

Fig. 4: (E) pill endometrium; (F) Endometritis; (G) Adenocarcinoma; (H) Squamous cell carcinoma

Table 1: Showing distribution of histopathological diagnosis of endometrial biopsies in different age groups

\begin{tabular}{|l|c|c|c|c|}
\hline & $<\mathbf{4 0}$ years & $\mathbf{4 0 - 5 0}$ years & $>\mathbf{5 0 y e a r s}$ & Total \\
\hline Proliferative phase & $108[27.0 \%]$ & $119[28.0 \%]$ & $32[18.2 \%]$ & $259[25.9 \%]$ \\
\hline Secretory phase & $130[32.5 \%]$ & $84[19.8 \%]$ & $10[5.68 \%]$ & $224[22.4 \%]$ \\
\hline Simple hyperplasia & $32[8.0 \%]$ & $59[13.9 \%]$ & $13[7.38 \%]$ & $104[10.4 \%]$ \\
\hline Complex hyperplasia & $11[2.75 \%]$ & $8[1.8 \%]$ & $24[13.6 \%]$ & $43[4.3 \%]$ \\
\hline Atrophic & 0 & $18[4.24 \%]$ & $51[28.9 \%]$ & $69[6.9 \%]$ \\
\hline Exogenous hormone & $54[13.5 \%]$ & $87[20.5 \%]$ & $7[3.97 \%]$ & $148[14.8 \%]$ \\
\hline Endometritis & $40[10.0 \%]$ & $19[4.4 \%]$ & 0 & $59[5.9 \%]$ \\
\hline Polyp & $21[5.25 \%]$ & $22[5.2 \%]$ & $28[15.9 \%]$ & $71[7.1 \%]$ \\
\hline Carcinoma & $4[1.0 \%]$ & $8[1.9 \%]$ & $11[6.25 \%]$ & $23[2.3 \%]$ \\
\hline Total & $400[\%]$ & $424[\%]$ & $176[100 \%]$ & $1000[\%]$ \\
\hline
\end{tabular}


Table 2: Table showing abnormal uterine bleeding and histopathological diagnosis of endometrial biopsy

\begin{tabular}{|c|c|c|c|c|c|c|c|c|}
\hline & Normal & Hyperplasia & Atrophic & $\begin{array}{c}\text { Exogenous } \\
\text { hormone }\end{array}$ & Endometritis & Polyp & Carcinoma & \\
\hline Polymenorrhoea & $105[51.4 \%]$ & $26[12.7 \%]$ & 0 & $33[16.1 \%]$ & $22[10.7 \%]$ & $17[8.33 \%]$ & $1[0.49 \%]$ & $204[100 \%]$ \\
\hline Oligomenorrhoea & $20[33.3 \%]$ & $2[3.33 \%]$ & $27[45 \%]$ & $4[6.66 \%]$ & $1[1.6 \%]$ & $6[10 \%]$ & 0 & $60[\%]$ \\
\hline Menorrhagia & $203[56.4 \%]$ & $33[9.2 \%]$ & $0[\%]$ & $62[17.2 \%]$ & $27[7.5 \%]$ & $31[8.6 \%]$ & $4[1.1 \%]$ & $360[100 \%]$ \\
\hline Metrorrhagia & $122[73.1 \%]$ & $9[5.38 \%]$ & 0 & $26[15.6 \%]$ & $3[1.8 \%]$ & $3[1.8 \%]$ & $4[2.4 \%]$ & $167[100 \%]$ \\
\hline Menometrorrhagia & $6[15.8 \%]$ & $4[10.5 \%]$ & \begin{tabular}{|l|}
0 \\
\end{tabular} & $17[44.7 \%]$ & $6[15.7 \%]$ & $2[5.2 \%]$ & $3[7.8 \%]$ & $38[100 \%]$ \\
\hline \multirow{2}{*}{$\begin{array}{l}\text { Post-menopausal } \\
\text { bleeding }\end{array}$} & $27[15.7 \%]$ & $73[42.7 \%]$ & $42[24.5 \%]$ & $6[3.5 \%]$ & 0 & $12[7.0 \%]$ & $11[6.4 \%]$ & $171[100 \%]$ \\
\hline & $483[48.3 \%]$ & $147[14.7 \%]$ & $69[6.9 \%]$ & $148[14.8 \%]$ & $59[5.9 \%]$ & $71[7.1 \%]$ & $23[2 . \%]$ & $1000[100 \%]$ \\
\hline
\end{tabular}

\section{Discussion}

Abnormal uterine bleeding is usually associated with hormonal disturbance or intrauterine pathology. The most common etiologies are structural uterine pathology (eg. Adenomyosis, fibroids, endometrial polyps) and anovulation, disorders of hemostasis and neoplasia. ${ }^{7}$ The present study showed that common cause for biopsy in our institute was menorrhagia in 360 [36.0\%] cases followed by polymenorrhea in 204 [20.4\%], post-menopausal bleeding 171[17.1\%], metrorrhagia 167 [16.7\%], oligomenorea 60 [6.0\%] and menometrorrhgia 38[3.8\%] cases. Juhi et al and Mohamed $\mathrm{AH}$ also found that, menorrhagia was the most prominent presenting symptom 57.4\% and 47\% cases, followed by postmenopausal bleeding $17.9 \%$ and $27.9 \%$ cases respectively. ${ }^{8,9}$ Whereas the study by Moghal $\mathrm{N}$ showed metrorrhagia as the main mode of presentation [48\%] followed by menorrhagia [41\%]. ${ }^{10}$ Also Abid et al study found that polymenorrhea was the most common pattern [30\%]. ${ }^{11}$

Our study shows that $25.9 \%$ of the biopsies revealed a proliferative phase endometrium and $22.4 \%$ showed secretory type of endometirum with no significant pathology. This is in accordance with high levels of oestrogen titre in blood which might give rise to such a picture; albeit hormonal imbalance itself can give rise to a different picture all together as described later. In our study maximum number of proliferative stage endometrium $(45.9 \%)$ were seen in $40-50$ age group followed by maximum number $(58 \%)$ of secretory phase endometrium in $<40$ years of age group. Similar to the study by Juhi et al, ${ }^{8}$ it is also to be noted that subjects of perimenopausal age group had predominance of proliferative endometrium whereas reproductive age group had more of secretory type endometrium. These studies are similar to that by Doraiswami $\mathrm{S}$ et $\mathrm{al}^{12}$ which showed maximum number of cases to be of proliferative and secretory phases which is also comparable to our study. In our study we found $147(14.7 \%)$ cases of hyperplasia. These findings are comparable to Doraiswami $\mathrm{S}$ et al. ${ }^{12}$ Similar findings were recorded by Mirza ${ }^{13}$ who found 35\% proliferative, $30 \%$ secretory and 30\% hyperplastic endometrium. Endometrial hyperplasia is quite common in perimenopausal women causing in majority of subjects, symptoms of irregular or prolonged bleeding due to anovulatory cycles. Heavy bleeding is secondary to sustained level of estrogens as stated earlier. The epithelial overgrowth affects glands and stroma and there is also abnormal vascularization. Bleeding is prolonged and excessive because of massive amount of hyperplastic tissue available for bleeding and random breakdown of tissue resulting in exposure of vascular channels.

Abnormalities along the hypothalamic-pituitaryovarian axis may result in derangements of follicular maturation, ovulation or corpus luteum formation resulting in changes in the hormonal milieu. These alterations in the normal hormonal patterns may lead to abnormal uterine bleeding. The incidence of secretory phase endometrium is slightly higher in perimenopausal women and this is consistent with the study by Jetley $\mathrm{S}$ et al. ${ }^{14}$

In our study, atrophic endometrium is seen in $28.9 \%$ of cases in $>50$ years age group. Perimenopausal age group eventually leads to permanent loss of ovarian function manifested as atrophic or inactive endometrium due anovulation lead to thinning of endometrium which easily bleeds on trauma. This is in concordance with the study by Anuradha et $\mathrm{al}^{15}$ and Abid $\mathrm{M}$ et $\mathrm{al}^{11}$ which showed $20 \%$ and $33 \%$ atrophic endometrium in post-menopausal age group respectively.

Complex hyperplasia in our study was more common in $>50$ years age group and presented mostly with irregular bleed. Simple hyperplasia was more common in the other two groups. This formed one of the most common lesion [21\%] accounting for endometrial bleeding in $>50$ years age group which is comparable 27\% and 30\% in Mirza et al and Pandey A et al study respectively. ${ }^{13,16}$ Endometrial hyperplasia is commonly seen in perimenopausal age group due to failure of ovulation. Persistent unripe follicles expose the endometrium to excessive and prolonged estrogenic action. This is slightly lower than the findings by Dangal G. ${ }^{17}$

A noticeable incidence of exogenous hormone endometrium or disordered proliferative endometrium [14.8\%] pattern was found in our study which is comparable to the Pandey A et al study and Doraiswami $\mathrm{S}$ et al. ${ }^{12,16}$ Similarly in our study endometritis is seen around $5.9 \%$ which is also 
comparable to $3.28 \%$ and $5.1 \%$ to studies done by Moghal and Doraiswami S et al respectively.

Both the incidence of polyp and carcinoma was much higher in post-menopausal age group and this is in concordance with the study by Pandey A et al and Mariam Abid et al. ${ }^{11,16}$ The overall incidence of endometrial carcinoma is $2.3 \%$ and this low incidence, when compared to studies of international regions, can be attributed to the early childbearing age and multiparity of women in Indian Subcontinent, which reduces the proliferative activity of endometrium. ${ }^{18}$

\section{Conclusion}

According to the findings of the present study, it can be concluded that, menorrhagia was the most common, followed by post-menopausal bleeding. It also showed that endometrial cause of AUB is age related pathology and the highest number of endometrial carcinoma tend to occur in post-menopausal age group and thus patients presenting with bleeding per vaginum in this age group needs to be evaluated thoroughly. Histopathological examination of endometrial biopsy is a major diagnostic tool in evaluation and a specific diagnosis could help the physician to plan therapy for successful management of AUB.

\section{Source of Support: Nil}

\section{Conflict of Interest: Nil}

\section{References}

1. Tavassoli FA, Devilee P; Pathology and genetics of tumours of the breast and female genital organs. Tumors of the uterine corpus. In WHO classifications of tumours. IARC Press, Lyon France, 2003:221-232.

2. Ely JW, Kennedy CM, Clark EC, Bowdler NC. Abnormal Uterine Bleeding: A Management Algorithm. J Am Board Fam Med. 2006;19:590-602.

3. Aksel S, Jones GS. Etiology and treatment of DUB. Obstet Gynecol. 1974; 44:1-13.

4. Altchek A. Dysfunctional uterine bleeding in adolescence. Clin Obstet Gynecol. 1977;20:633-650.

5. Padubidri VG, Daftary SN. Howkins and Bourne Shaw's textbook of gynaecology.16 $6^{\text {th }}$ ed. Elsevier;2015:321-22.

6. Koss LG, Durfee GR: Cytologic diagnosis of endometrial carcinoma. Results of ten years of experience. Acta Cytol. 1962,6:519-31.

7. Sudha, Pallavi. Distribution of Causes of AUB According to PALM-COEIN Classification in a Tertiary Care Center, International Journal of Scientific Study. 2017;4;10:160-163.

8. Juhi S, Ruchika B. A study of spectrum of morphological changes in endometrium in abnormal uterine bleeding. Journal of advance researches in biological sciences. 2013;5(4):370-375.

9. Mohamed A H. Abnormal Uterine Bleeding and its Impact on Women Life. IOSR Journal of Nursing and Health Science (IOSR-JNHS), 2017;6(5):30-37.

10. Moghal N. Diagnostic value of endometrial curettage in abnormal uterine bleeding-a histopathologic study. J Pak Med Assoc. 1997;47(12):295-9.

11. Abid M, Hashmi AA, Malik B. Clinical pattern and spectrum of endometrial pathologies in patients with abnormal uterine bleeding in Pakistan: need to adopt a more conservative approach to treatment. BMC Women's Health. 2014;14:132. doi:10.1186/s12905-014-0132-7.

12. Doraiswami S, Johnson T, Rao S, Rajkumar A, Vijayaraghavan J, Panicker VK. Study of endometrial pathology in abnormal uterine bleeding. J Obstet Gynecol. 2011;61(4):426-30.

13. Mirza T, Akram S, Mirza A, Aziz S, Mirza T, Mustansar T. Histopathological pattern of abnormal uterine bleeding in endometrial biopsies. Journal of Basic \& Applied Sciences. 2012;8:114-17. DOI:

http://dx.doi.org/10.6000/19275129.2012.08.01.2

14. Jairajpuri Z, Jetley S, Rana, S. Morphological spectrum of endometrial pathology in middle-aged women with atypical uterine bleeding: A study of 219 cases. Journal of Mid-life Health. 2013;4(4):216.

15. Anuradha, S. and Premlata, M. Spectrum of Endometrial Histopathology in Women Presenting with Abnormal Uterine Bleeding. Scholars Journal of Applied Medical Sciences. 2015;3(1):1-4.

16. Padhye A, Kaul U, Dhar R Histopathology of Endometrial Biopsies in Cases of Abnormal Uterine Bleeding- A Four Year Study JMSCR. 2017;05;05:21597-21599

17. Dangal G. A study of endometrium in patients with abnormal uterine bleeding at Chitwan valley. Kathmandu University Medical Journal. 2003;1(2):110-112.

18. Cramer DW. The Epidemiology of Endometrial and Ovarian Cancer. Hematology/Oncology Clinics of North America. 2012;26(1):1-12. doi:10.1016/j.hoc.2011.10.009.

How to cite this article: Iqbal M. B, Kambale T, Khandelwal A, Koshy A, Banerjee B. Spectrum of Endometrial lesions in patients presenting with abnormal uterine bleeding. Indian J Pathol Oncol. 2018;5(4):587-591. 